\title{
Chercheurs \\ du monde entier, \\ unissez-vous !
}

La science

à bout de souffle?

Laurent Ségalat

Hélène Gilgenkrantz

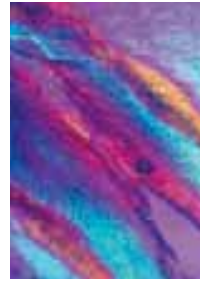

\author{
H. Gilgenkrantz : \\ Inserm U567-UMR CNRS 8104, \\ Institut Cochin, \\ 24, rue du Faubourg \\ Saint-Jacques, \\ 75014 Paris, France. \\ helene.gilgenkrantz@inserm.fr
}

À l'heure où le ministère vient d'instaurer des primes au mérite uniquement dévolues aux chercheurs ayant déjà bénéficié de prix essentiellement internationaux, Laurent Ségalat tire la sonnette d'alarme: le système scientifique est proche du point de rupture... La science est à bout de souffle [1]. Le constat est sans appel et ne s'arrête pas aux frontières des spécificités françaises. C'est un pamphlet mondialisé qui nous rappelle que «dans un système qui mélange les notions de succès et de mérite, la sagesse prévaut rarement ». Commençons par la fin: le parallélisme entre science et finance. Le dogme dominant en matière d'économie fut en effet longtemps que les marchés se régulaient eux-mêmes avec cette idée darwinienne que la sélection naturelle améliorait la compétitivité. Le résultat est devant nous, les bulles financières ont explosé nous laissant au milieu d'une crise que d'aucuns disaient prévisible alors que l'histoire semble ne pas avoir de mémoire. Il en est de la recherche comme de l'économie et Laurent Ségalat nous suggère alors de «lire Darwin jusqu'au bout ». En effet, la compétition fait partie intégrante du métier de chercheur. Elle lui est bénéfique et stimule son efficacité à apporter des connaissances. Dans la théorie darwinienne, cette pression continue et positive aboutit à une sélection des espèces à leur environnement tout en préservant leur biodiversité. Mais qu'advient-il quand le système est poussé à l'excès, quand la pression devient trop forte? Elle induit une sélection plus pointue aux dépens de la diversité. Le scientifique serait-il une espèce en voie de disparition? Probablement pas, mais cette évolution fait planer le spectre du scientifique formaté, du chercheur unique. Si ce nouveau chercheur, devenu surtout manager, est certes mieux adapté aux demandes itératives de financement et totalement aguerri aux impact factor des journaux et autre calcul de facteur h, est-il pour autant un meilleur producteur de connaissances? II n'a, en tout cas, plus le temps d'être rigoureux, créatif ou de remettre en question les dogmes acquis. En matière de production de connaissances, «rentabilité » est donc antinomique de «découverte». Elle annihile toute promesse d'inattendu. Elle est l'opposé de la sélection darwinienne naturelle qui tient sa richesse de ce qu'elle n'est pas prédéfinie, pas prévisible ni téléologique, alors que la sélection actuelle des chercheurs s'achemine vers une reproduction à l'identique d'un modèle unique et formaté. En quelques citations savoureuses et quelques comparaisons piquantes, Laurent Ségalat nous rappelle ce que notre système actuel de financement et d'évaluation de la recherche a d'absurde alors que la «science a besoin de temps, de stabilité, de 
chercheurs qui creusent patiemment leurs sillons, de créativité, de prise de risque ». L'état des lieux est à peine exagéré.

Quelles solutions apporter si le navire prend déjà l'eau, si la pandémie est mondiale? Puisque le malade est mourant, les remèdes se devraient d'être administrés rapidement. Malheureusement, l'ouvrage de Laurent Ségalat n'a d'autres armes que de suggérer aux scientifiques de se réapproprier la valeur des travaux collaboratifs, notamment dans des compétences similaires et de ralentir la cadence des temps modernes au profit d'une réflexion globale et constructive.

Alors, pour paraphraser une des citations du livre émanant d'un récent prix Nobel : puisque «en sciences, le paysage final n'est jamais visible du point de départ », espérons que le diagnostic était un peu alarmiste et que cet électrochoc aura alors le mérite de nous réorienter vers un futur plus serein et enthousiaste. Reste que, comme dans une chanson de Paco Ibanez: "Perdóname no sé decirte nada más, pero tú comprende que yo aún estoy en el camino ${ }^{1}$... Mais le temps de la lecture de ce livre bien écrit et non dénué d'humour, je me suis sentie moins seule sur ce chemin. $\diamond$

The science out of breath

\section{CONFLIT D'INTÉRÊTS}

L'auteur déclare n'avoir aucun conflit d'intérêts concernant les données publiées dans cet article.

\section{RÉFÉRENCE}

1. Ségalat L. La science à bout de souffle? Paris : Seuil, $2009: 108$ p.

${ }^{1}$ «Pardonne moi, mais je ne peux t'en dire plus, car tu comprendras que, moi aussi, je suis sur le chemin », tiré d'une chanson de Paco Ibanez: Palabras para Julia.

\section{TIRÉS À PART}

H. Gilgenkrantz

\section{Bon de commande}

À retourner à EDK, 2, rue Troyon - 92316 Sèvres Cedex

Tél. : 0155641393 - Fax : 0155641394 - E-mail : edk@edk.fr

NOM : Prénom :

Adresse :

Code postal :

Ville :

Pays :

Fonction :

Je souhaite recevoir l'ouvrage Panim/Pnim, l'exil prend-il au visage $?: 25 €+3 €$ de port $=\mathbf{2 8} €$ TTC

en ................ exemplaire, soit un total de ................................... €

$\square$ Par chèque, à l'ordre de $\mathbf{E} \mathbf{D} \mathbf{K}$

$\square$ Par carte bancaire : $\square$ Visa $\square$ Eurocard/Mastercard

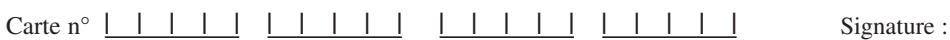

Date d'expiration: $\quad|\quad| \quad|\quad|$

$\mathrm{N}^{\circ}$ de contrôle au dos de la carte : $\quad \underline{|\quad| \quad \mid}$

ISBN : 978-2-8425-4135-4 392 pages

\section{Bon de commande}

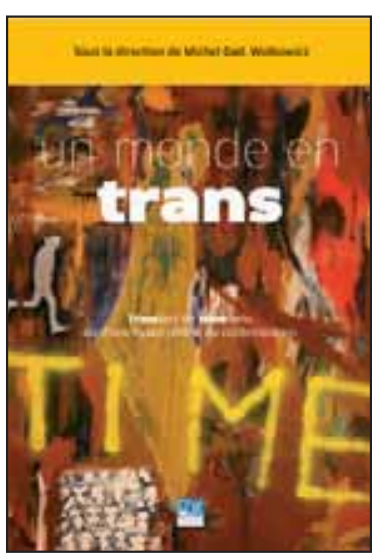

ISBN : 978-2-8425-4129-3 344 pages
À retourner à EDK, 2, rue Troyon - 92316 Sèvres Cedex

Tél. : 0155641393 - Fax : 0155641394 - E-mail : edk@edk.fr

NOM : . P Prénom :

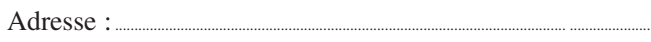

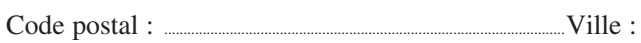

Pays :

Fonction :

Je souhaite recevoir l'ouvrage Un monde en trans : $24 €+3 €$ de port $=27 €$ TTC

en ................ exemplaire, soit un total de ..................................... €

$\square$ Par chèque, à l'ordre de $\mathbf{E} \mathbf{D} \mathbf{K}$

$\square$ Par carte bancaire : $\square$ Visa $\square$ Eurocard/Mastercard

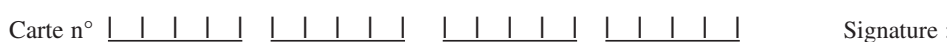

Date d'expiration: $\quad \underline{1 \mid} \underline{1||}$

$\mathrm{N}^{\circ}$ de contrôle au dos de la carte : $\quad|\quad| \quad|\quad|$ 